\title{
Sinusoidal heart rate rhythms in severe neonatal hypoxia
}

\author{
MARK McC. REID, J. JENKINS, AND GARTH McCLURE \\ Special Care Baby Unit, Royal Maternity Hospital, and Jubilee Maternity Hospital, and \\ Department of Child Health, Queen's University, Belfast
}

SUMMARY Sinusoidal heart rate traces were detected in 8 patients, 6 of whom died; the 2 survivors showed evidence of gross cerebral damage. It is felt that the origin of the sinusoidal curve is probably central and reflects loss of central control of heart rate.

Continuous monitoring of the fetal heart rate through labour has led to the detection of abnormal heart rate patterns indicative of fetal distress. One significant pattern detected by Baskett and Ko (1974) and Manseau et al. (1972) was the sinusoidal heart rate pattern, characterised by slow regular wave forms in the fetal heart rate. This pattern in the neonatal period was also described by Ekert and Schenk (1974). In all reports the appearance of this pattern was associated with a poor prognosis. We wish to report our observations on sinusoidal heart rate patterns seen in 8 infants in a one-year period in two special care baby units in Belfast.

\section{Patients and methods}

During a period of one year all ill newborn infants receiving intensive care in our units in Belfast were monitored using cardiorespirographs (Corometrics 512 and Hewlett-Packard Cardio-Respiratory Monitor 78250A). These monitors detect successive $\mathrm{R}$-waves on the electrocardiograph and give an instantaneous heart rate, both as a digital read-out and on a paper trace. The respiratory rate and amplitude are detected, by an impedance method, by two electrodes placed laterally on the chest wall.

During the period under review 130 infants were monitored. The infants suffered from a variety of conditions including idiopathic respiratory distress syndrome (IRDS), severe congenital heart disease, rhesus haemolytic disease, and apnoeic spells of prematurity. The clinical data of the 8 patients with sinusoidal heart rate traces are given below.

Royal Maternity Hospital, Belfast

MARK MCC. REID, consultant paediatrician

GARTH MCCLURE, consultant paediatrician

J. JENKINS, research fellow
Case 1. A baby boy delivered by caesarean section in a peripheral hospital at $\mathbf{4 3}$ weeks' gestation with a birthweight of $4600 \mathrm{~g}$. Caesarean section was performed because of delay in labour which had lasted 36 hours. Meconium-stained liquor had been passed and fetal bradycardia had occurred intermittently in the 24 hours before delivery. The Apgar score was 2 at five minutes and spontaneous respirations were not attained until age 10 minutes. The infant then developed a series of apnoeic spells and was transferred at age 6 hours. On admission the infant was hypotonic and had periodic apnoeic spells. Six hours later convulsions developed which were then treated with intravenous diazepam. In spite of this the infant developed further convulsions and became increasingly hypertonic and died at age $3 \frac{1}{2}$ days. Necropsy showed cerebral softening and oedema but with no haemorrhage or significant abnormalities of other organs.

Case 2. A baby boy with a birthweight of $3600 \mathrm{~g}$, delivered by forceps in a peripheral hospital. The Apgar score was 3 at five minutes and regular respirations were not established until 45 minutes despite active resuscitation. The infant was transferred and admitted to our unit at age 11 hours. At that time he was shocked, cyanosed, and hypoventilating. He was hypotonic and developed increasingly frequent apnoeic spells and died at age 2 days. Necropsy showed cerebral oedema and also subarachnoid, subdural, and pulmonary haemorrhage.

Case 3. A baby girl weighing $3500 \mathrm{~g}$. Delivery was by Kielland's forceps for occipito-posterior position and abruptio placenta at term. Apgar scores were 2 and 4 at two and five minutes respectively. Active resuscitation was carried out but spontaneous 
respirations were not attained until 30 minutes. During the next 24 hours the baby became hypertonic and developed convulsions associated with bradycardia, apnoea, and cyanotic attacks. CSF was evenly blood stained. Spontaneous recovery occurred and the infant survived but suffers from microcephaly, spastic quadriplegia, salaam attacks, and gross developmental delay.

Case 4. A baby boy weighing $2900 \mathrm{~g}$ at term by normal delivery. Fetal distress had occurred during labour and the infant's Apgar scores were 4 and 2 at two and five minutes. During the first 4 days of life he was deeply unconscious and suffered from convulsive episodes and occasional apnoeic spells. Tone was variable, being initially hypotonic and then hypertonic. The infant survived but is now microcephalic with salaam spasms and pronounced hypotonia. There is also gross developmental delay.

Case 5. A baby boy born at 35 weeks' gestation by normal delivery, with a birthweight of $2700 \mathrm{~g}$ after spontaneous onset of preterm labour in a peripheral hospital. The infant's condition was satisfactory at birth but he then developed IRDS which was confirmed by chest $x$-ray. He was therefore transferred to one of our units at 20 hours of age. On examination he was severely ill, and cyanosed in $100 \%$ oxygen with an arterial $\mathrm{PO}_{2}$ of 17 . The $\mathrm{pH}$ was 6.89 and $\mathrm{PCO}_{2}$ was 132 . The infant was intubated and received intermittent positive pressure ventilation (IPPV), but died one hour later aged 21 hours. Necropsy showed evidence of pronounced pulmonary atelectasis and also subarachnoid haemorrhage.

Case 6. A baby girl born by normal delivery at 29 weeks' gestation, birthweight $1300 \mathrm{~g}$, after spontaneous onset of labour. Apgar scores were 1 at five and 2 at ten minutes. In spite of active resuscitation the infant remained very ill with intermittent apnoeic spells and convulsions. CSF was evenly blood stained and the fontanelles were distended and tense, suggesting intracranial haemorrhage. The infant developed bronchopneumonia and died aged 4 days. Unfortunately consent for necropsy could not be obtained.

Case 7. A baby girl born by assisted breech delivery at 29 weeks' gestation with a weight of $1130 \mathrm{~g}$ after spontaneous onset of preterm labour. Apgar score was 4 at two minutes and the baby rapidly developed respiratory distress. On admission to the special care baby unit she was noted to be anaemic with a haematocrit of 40 . The infant was treated with IPPV, blood transfusion, and correction of acidosis, but she failed to respond to treatment and died aged 6 hours 20 minutes. Necropsy showed a small intraventricular haemorrhage and pronounced pulmonary atelectasis.

Case 8. A baby boy delivered at 28 weeks' gestation after antepartum haemorrhage and breech delivery. The birthweight was $1080 \mathrm{~g}$, Apgar score was 1 at two minutes. Spontaneous respirations were not established and the baby was treated with IPPV; however, he failed to respond to treatment and died aged 18 hours. Necropsy showed pronounced pulmonary atelectasis, a small pulmonary haemorrhage, and subarachnoid haemorrhage.

These clinical features together with a description of the sinusoidal patterns are shown in the Table.

All 8 infants demonstrated a sinusoidal heart rate. Cases 4 and 8 (Figs 1 and 2) are shown as examples. The frequency of the sinusoidal curve varied in the infants from once every 30 seconds to once every

Table Clinical features and sinusoidal heart rate patterns in 8 infants

\begin{tabular}{|c|c|c|c|c|c|c|}
\hline \multirow[t]{2}{*}{ Case } & \multirow{2}{*}{$\begin{array}{l}\text { Weight } \\
(g)\end{array}$} & \multirow[t]{2}{*}{ Diagnosis } & \multicolumn{3}{|c|}{ Sinusoidal pattern } & \multirow[t]{2}{*}{ Outcome } \\
\hline & & & Duration & $\begin{array}{l}\text { Frequency } \\
\text { (min) }\end{array}$ & Rate/min & \\
\hline 1 & 4600 & Asphyxia & $3 \mathrm{~h}$ & $3 \frac{1}{2}$ & $110-130$ & $\begin{array}{l}\text { Died } 3 \frac{1}{2} \text { days. Necropsy: cerebral oedema } \\
\text { and softening }\end{array}$ \\
\hline 2 & 3600 & Asphyxia & $40 \mathrm{~min}$ & $2 \frac{1}{2}$ & $120-180$ & $\begin{array}{l}\text { Died } 2 \text { days. Necropsy: subarachnoid and } \\
\text { subdural haemorrhage }\end{array}$ \\
\hline 3 & 3500 & Asphyxia & $4 \mathrm{~h}$ & 1 & $140-150$ & $\begin{array}{l}\text { Lived. Microcephaly, epilepsy, and } \\
\text { spastic quadriplegia }\end{array}$ \\
\hline 4 & 2900 & Asphyxia & $48 \mathrm{~h}$ & $1 \frac{1}{2}$ & $90-120$ & $\begin{array}{l}\text { Lived. Microcephaly, epilepsy, and } \\
\text { hypotonia }\end{array}$ \\
\hline 5 & 2740 & IRDS & $8 \mathrm{~min}$ & 1 & $110-150$ & $\begin{array}{l}\text { Died } 21 \text { hours. Necropsy: atelectasis, } \\
\text { subarachnoid haemorrhage }\end{array}$ \\
\hline 6 & 1300 & Asphyxia, bronchopneumonia & $12 \mathrm{~h}$ & $\frac{1}{2}$ & $130-150$ & $\begin{array}{l}\text { Died } 4 \text { days. No necropsy. Intraventricular } \\
\text { haemorrhage and bronchopneumonia }\end{array}$ \\
\hline 7 & 1130 & Asphyxia, IRDS & $35 \mathrm{~min}$ & 1 & $60-120$ & $\begin{array}{l}\text { Died } 6 \text { hours. Necropsy: atelectasis, } \\
\text { intraventricular haemorrhage }\end{array}$ \\
\hline 8 & 1080 & Asphyxia, IRDS & $8 \mathrm{~min}$ & 11 & $105-140$ & $\begin{array}{l}\text { Died } 18 \text { hours. Necropsy: atelectasis, } \\
\text { subarachnoid haemorrhage }\end{array}$ \\
\hline
\end{tabular}


$3 \frac{1}{2}$ minutes and the amplitude from 10 to 60 beats/ min. None of the remaining 122 infants who were monitored showed sinusoidal heart rate rhythms. 14 of these infants died, of whom 4 showed evidence of cerebral disease at necropsy but had not exhibited the sinusoidal curve patterns during life. The remaining infants died of extreme prematurity, with or without IRDS, or from congenital malformations. Of the 108 babies who were monitored and survived and who did not show sinusoidal heart rate patterns, none to date has shown evidence of neurological impairment although the follow-up period has been short, most of these infants still being under one year.

\section{Discussion}

Sinusoidal heart rate rhythms can clearly be distinguished from other heart rate patterns seen in the neonatal period as Figs 3 and 4 clearly show. Previous authors have noted that this pattern is associated with a grave prognosis, and all 8
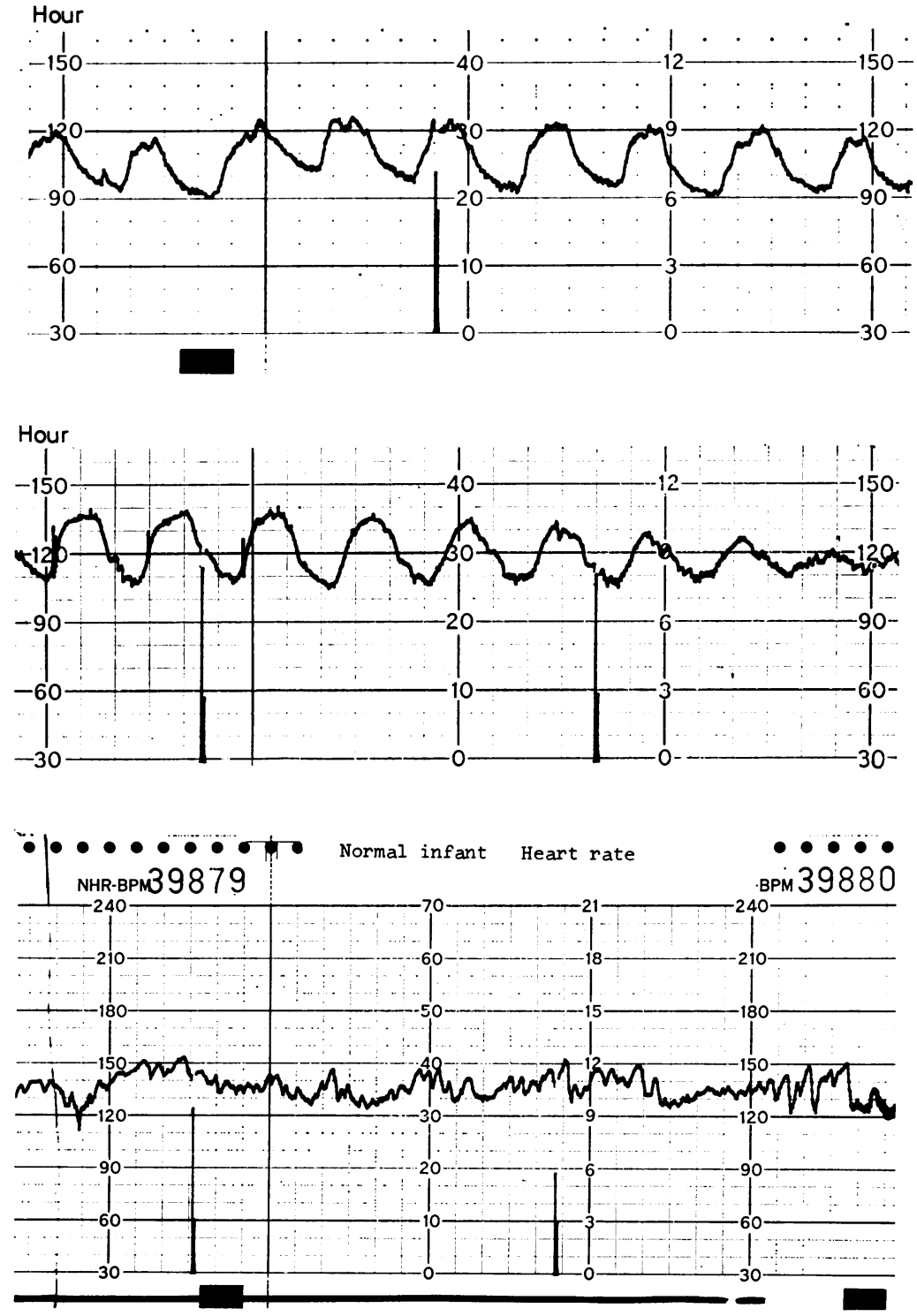

Fig. 1 Vertical axis heart rate. Horizontally each major dark line represents a 6 min-interval.

Fig. 2 Vertical axis heart rate. Horizontally each major dark line represents a 6 min-interval.

Fig. 3 Normal infant, normal heart rate pattern. 


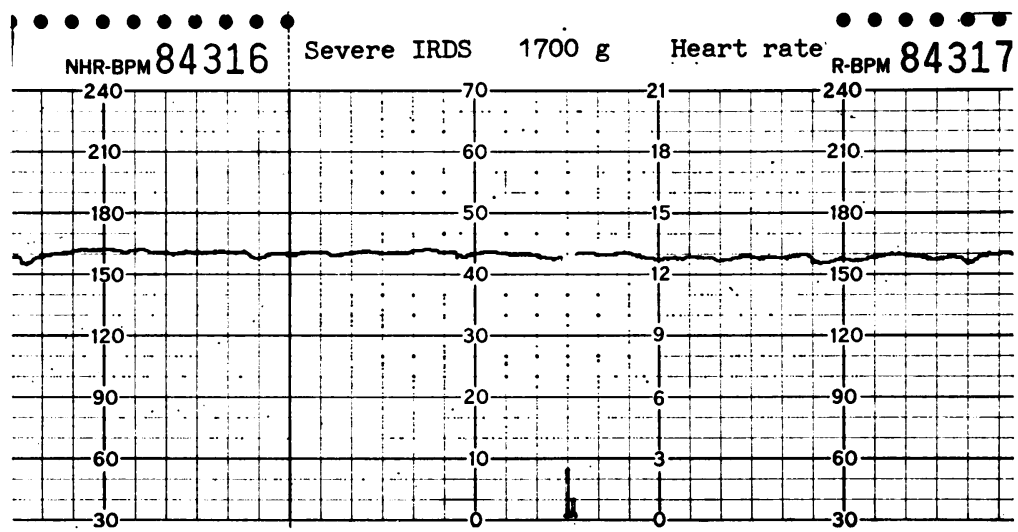

Fig. 4 Heart rate 'flat'. Heart rate trace in an infant with severe IRDS.

infants in whom we have seen this pattern have either died or have had gross residual cerebral damage. Other heart rate abnormalities, such as decreased heart rate variation, have been seen in children with brain damage and it is claimed that it may be considered a clinical criterion of brain death (Kero et al., 1978). However, it must be stressed that the total observation of the sinusoidal heart rate pattern is extremely limited and many further observations would be needed to confirm its prognostic significance.

The origin of the sinusoidal curve is in some doubt. Heart rate deceleration may occur in response to a primary respiratory event as we would expect in the current apnoeic episodes. Valbona et al. (1965) studied adults with decerebrate states and described similar patterns with prominent waves of acceleration of heart rate rhythm followed by rebound deceleration. These waves coincided with each deep breath or with the onset of periodic breathing. Guilleminault et al. (1977) described cardiac arrhythmias in adults with apnoeic spells and severe CNS disease. In several of our patients the sinusoidal wave pattern was associated with recurrent regular apnoeic spells. However, in our cases there was no fixed relationship between the occurrence of apnoea and cardiac deceleration, as 3 of our 8 patients were receiving IPPV at the time when the sinusoidal heart rate pattern was seen. We feel that in these 3 cases at least, the sinusoidal heart rate pattern is unlikely to be a primary respiratory event and is more probably central in origin and may reflect a lack of central control of cardiac rhythm.

Sinusoidal patterns could also possibly be a primary cardiac phenomenon reflecting either conduction defects or a diseased myocardium. Cardiac arrhythmias have been reported in fetuses and infants during hypoxic stress but unfortunately in none of our patients did we obtain either direct electrocardiographic traces or cardiac enzyme studies.
These patterns appear to have a grave prognosis, but as there is little experience we would urge caution in their interpretation, and we hope that there will be many further observations before any conclusions are made and the patients treated accordingly.

We thank all the junior medical staff and nursing staff for their careful record keeping and assistance in the collection of these records. J.J. is in receipt of a fellowship from DHSS, NI.

\section{References}

Baskett, T. K., and Ko, K. S. (1974). Sinusoidal fetal heart patterns. A sign of fetal hypoxia. Obstetrics and Gynecology, 44, 379-382.

Ekert, W. N., and Schenk, H. W. (1974). Findings from monitoring premature and full-term newborns with the cardio-respirograph. In Neonatal Monitoring Cardiorespirography. Proceedings of the Seventh German Congress on Perinatal Medicine Berlin, 13-17 November 1974.

Guilleminault, C., Tilkian, A., Lehrman, K., Forno, L., and Dement, W. C. (1977). Sleep apnoea syndrome. States of sleep and autonomic dysfunction. Journal of Neurology, Neurosurgery, and Psychiatry, 40, 718-725.

Kero, P., Antila, K., Ylitalo, V., and Välimäki, I. (1978). Decreased heart rate variation in decerebration syndrome: quantitative clinical criterion of brain death? Pediatrics, 62, 307-311.

Manseau, P., Vanquier, J., Chavinié, J., and Sureou, C. (1972). Le rhythme cardiaque foetal 'sinusoidal'. Aspect évocateur de souffrance foetale au cours de la grossesse. Journal de gynécologie obstétrique et biologie de la réproduction, 1, 343-352.

Valbona, V., Cardus, D., Spencer, W. A., and Hoff, H. E. (1965). Patterns of sinus arrhythmia in patients with lesions of the central nervous system. American Journal of Cardiology, 16, 379-389.

Correspondence to Dr M. McC. Reid, Royal Maternity Hospital, Belfast B12 6BB, Northern Ireland.

Received 7 November 1978 